\title{
THE GREEDY INDEPENDENT SET IN A RANDOM GRAPH WITH GIVEN DEGREES
}

\author{
GRAHAM BRIGHTWELL, SVANTE JANSON, AND MALWINA LUCZAK
}

\begin{abstract}
We analyse the size of an independent set in a random graph on $n$ vertices with specified vertex degrees, constructed via a simple greedy algorithm: order the vertices arbitrarily, and, for each vertex in turn, place it in the independent set unless it is adjacent to some vertex already chosen. We find the limit of the expected proportion of vertices in the greedy independent set as $n \rightarrow \infty$ (the jamming constant), expressed as an integral whose upper limit is defined implicitly, valid whenever the second moment of a random vertex degree is uniformly bounded. We further show that the random proportion of vertices in the independent set converges in probability to the jamming constant as $n \rightarrow \infty$. The results hold under weaker assumptions in a random multigraph with given degrees constructed via the configuration model.
\end{abstract}

\section{INTRODUCTION}

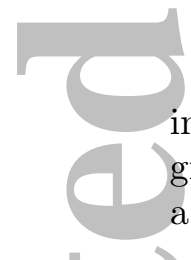

In this paper, we analyse a simple greedy algorithm for constructing an independent set in a random graph chosen uniformly from those with a given degree sequence, under some mild regularity assumptions. We obtain a nearly explicit formula for the size of the independent set constructed.

Our method involves generating the random graph via the configuration model, simultaneously with running the greedy algorithm. In the present context, this idea was first used by Wormald [27], who treated the special case of random regular graphs. Our methods follow those developed in a sequence of papers by Janson and Luczak [18; 19], and most particularly a recent paper of Janson, Luczak and Windridge [20] (see the proof of Theorem 2.6(c) in that paper).

We consider the following natural greedy process for generating an independent set $\mathcal{S}$ in an $n$-vertex (multi)graph. We start with $\mathcal{S}$ empty, and we consider the vertices one by one, in a uniformly random order. At each step, if the vertex under consideration is not adjacent to a vertex in $\mathcal{S}$, we put it

\footnotetext{
Date: 18 August 2016.

2000 Mathematics Subject Classification. 60J27, 92D30.

Key words and phrases. greedy independent set; jamming constant; configuration model.

Svante Janson is supported by the Knut and Alice Wallenberg Foundation.

The research of Malwina Luczak is supported by an EPSRC Leadership Fellowship, grant reference $\mathrm{EP} / \mathrm{J} 004022 / 2$.
}

1

This is the author manuscript accepted for publication and has undergone full peer review but has not been through the copyediting, typesetting, pagination and proofreading process, which may lead to differences between this version and the Version record. Please cite this article as doi:10.1002/ rsa.20716. 
in $\mathcal{S}$, and otherwise we do nothing. The set $\mathcal{S}$ at the end of the process is sometimes called the greedy independent set or jamming limit; let $S_{\infty}=S_{\infty}^{(n)}$ be the size of the greedy independent set. (Note: a multigraph may have loops, and it might be thought natural to exclude a looped vertex from the independent set, but as a matter of convenience we do not do this, and we allow looped vertices into our independent set. Ultimately, the main interest is in the case of graphs.)

For $n \in \mathbb{N}$ and a sequence $\left(d_{i}\right)_{1}^{n}$ of non-negative integers, let $G\left(n,\left(d_{i}\right)_{1}^{n}\right)$ be a simple graph (i.e. with no loops or multiple edges) on $n$ vertices chosen uniformly at random from among all graphs with degree sequence $\left(d_{i}\right)_{1}^{n}$. (We tacitly assume there is some such graph, so $\sum_{i=1}^{n} d_{i}$ must be even, at least.) We let $G^{*}\left(n,\left(d_{i}\right)_{1}^{n}\right)$ be the random multigraph with given degree sequence $\left(d_{i}\right)_{1}^{n}$ defined by the configuration model, first introduced in Bollobás [3]. That is, we take a set of $d_{i}$ half-edges for each vertex $i$ and combine the half-edges into pairs by a uniformly random matching of the set of all halfedges. Conditioned on the multigraph being a (simple) graph, we obtain $G\left(n,\left(d_{i}\right)_{1}^{n}\right)$, the uniformly distributed random graph with the given degree sequence.

Let

$$
n_{k}=n_{k}(n)=\#\left\{i: d_{i}=k\right\}, \quad k \in \mathbb{Z}^{+}:=\{0,1,2, \ldots\},
$$

the number of vertices of degree $k$ in $G\left(n,\left(d_{i}\right)_{1}^{n}\right)$ (or $G^{*}\left(n,\left(d_{i}\right)_{1}^{n}\right)$ ). Then $\sum_{k} n_{k}=n$ and $\sum_{k} k n_{k}$ is even.

Let $\left(p_{k}\right)_{0}^{\infty}$ be a probability distribution, and assume that $n_{k} / n \rightarrow p_{k}$ for each $k \in \mathbb{Z}^{+}$. We assume further that the distribution $\left(p_{k}\right)_{0}^{\infty}$ has a finite and positive mean $\lambda=\sum_{k=1}^{\infty} k p_{k}$ and that the average vertex degree $\sum_{k} k n_{k} / n$ converges to $\lambda$. (Equivalently, the distribution of the degree of a random vertex is uniformly integrable. In Section 5 , we consider the size of a greedy independent set in a random multigraph when we relax this condition.)

We prove our results for the greedy independent set process on $G^{*}$, and, by conditioning on $G^{*}$ being simple, we deduce that these results also hold for the greedy independent set process on $G$. For this, we use a standard argument that relies on the probability that $G^{*}$ is simple being bounded away from zero as $n \rightarrow \infty$. By the main theorem of [16] (see also [17]) this occurs if and only if $\sum_{k=1}^{\infty} k^{2} n_{k}(n)=O(n)$. (Equivalently, the second moment of the degree distribution of a random vertex is uniformly bounded.)

Our aim in this paper is to show that, under the conditions above, the expected proportion of vertices in the greedy independent set converges to a limit as $n$ tends to infinity (sometimes called the jamming constant) and to give an expression for the jamming constant in terms of the $p_{k}$. We also show that the size of the random greedy independent set in $G\left(n,\left(d_{i}\right)_{1}^{n}\right)$ or $G^{*}\left(n,\left(d_{i}\right)_{1}^{n}\right)$, divided by $n$, converges to the jamming constant in probability as $n$ tends to infinity. 
Theorem 1.1. Let $\left(p_{k}\right)_{0}^{\infty}$ be a probability distribution, and let $\lambda=\sum_{k=1}^{\infty} k p_{k} \in$ $(0, \infty)$. Assume that $n_{k} / n \rightarrow p_{k}$ for each $k \in \mathbb{Z}^{+}$and that $\sum_{k=1}^{\infty} k n_{k} / n \rightarrow \lambda$ as $n \rightarrow \infty$.

Let $S_{\infty}^{(n)}$ denote the size of a random greedy independent set in the random multigraph $G^{*}\left(n,\left(d_{i}\right)_{1}^{n}\right)$.

Let $\tau_{\infty}$ be the unique value in $(0, \infty]$ such that

$$
\begin{gathered}
\lambda \int_{0}^{\tau_{\infty}} \frac{e^{-2 \sigma}}{\sum_{k} k p_{k} e^{-k \sigma}} d \sigma=1 . \\
\frac{S_{\infty}^{(n)}}{n} \rightarrow \lambda \int_{0}^{\tau_{\infty}} e^{-2 \sigma} \frac{\sum_{k} p_{k} e^{-k \sigma}}{\sum_{k} k p_{k} e^{-k \sigma}} d \sigma \quad \text { in probability. }
\end{gathered}
$$

Then$$
\left(\frac{1}{2}\right)
$$

The same holds if $S_{\infty}^{(n)}$ is the size of a random greedy independent set in the random graph $G\left(n,\left(d_{i}\right)_{1}^{n}\right)$, if we assume additionally that $\sum_{k=1}^{\infty} k^{2} n_{k}=O(n)$ as $n \rightarrow \infty$.

Since $S_{\infty}^{(n)} / n$ is bounded (by 1 ), it follows that the expectation $\mathbb{E} S_{\infty}^{(n)} / n$ also tends to the limit in (1.3) under the hypotheses in the theorem.

Our proof yields also the asymptotic degree distribution in the random greedy independent set.

Theorem 1.2. Under the assumptions of Theorem 1.1, let $S_{\infty}^{(n)}(k)$ denote the number of vertices of degree $k$ in the random greedy independent set in the random multigraph $G^{*}\left(n,\left(d_{i}\right)_{1}^{n}\right)$. Then, for each $k=0,1, \ldots$,

$$
\frac{S_{\infty}^{(n)}(k)}{n} \rightarrow \lambda \int_{0}^{\tau_{\infty}} e^{-2 \sigma} \frac{p_{k} e^{-k \sigma}}{\sum_{j} j p_{j} e^{-j \sigma}} d \sigma \quad \text { in probability. }
$$

The same holds in the random graph $G\left(n,\left(d_{i}\right)_{1}^{n}\right)$, if we assume additionally that $\sum_{k=1}^{\infty} k^{2} n_{k}=O(n)$ as $n \rightarrow \infty$.

Remark 1.3. We do not know whether the theorems hold also for the simple random graph $G\left(n,\left(d_{i}\right)_{1}^{n}\right)$ without the additional hypothesis that $\sum_{k} k^{2} n_{k}=O(n)$. We leave this as an open problem. (See Example 5.5 for a counterexample if we do not even assume that $k n_{k} / n$ is uniformly summable.)

One natural special case concerns a random $d$-regular graph, which is covered by the case where $p_{d}=1$, for some $d$. It is not hard to see that the jamming constant of a random 2-regular graph, in the limit as $n \rightarrow \infty$, is the same as that of a single cycle (or path), again in the limit as the number of vertices tends to infinity. An equivalent version of the greedy process in this case is for "cars" to arrive sequentially, choose some pair of adjacent vertices on the cycle, and occupy both if they are both currently empty. This is then a discrete variant of the Rényi parking problem [26]. The limiting density of occupied vertices was first calculated by Flory [11] in 1939 to be $\frac{1}{2}\left(1-e^{-2}\right)$. See also Page [24], Evans [8] and Gerin [15]. 
The case of random $d$-regular graphs for $d \geq 3$ was first treated by Wormald [27], in a 1995 paper where he first gives a general scheme for his differential equations method, and independently by Frieze and Suen [13] for $d=3$. Wormald gives an analysis of exactly the random process we consider, in the special case of $d$-regular graphs, and obtains the result that the greedy independent set is, w.h.p. (i.e., with probability tending to 1 as $n \rightarrow \infty)$, of size

$$
\frac{n}{2}\left(1-\left(\frac{1}{d-1}\right)^{2 /(d-2)}\right)+o(n) .
$$

We shall verify shortly that our results give the formulae above in these cases. Lauer and Wormald [22], and Gamarnik and Goldberg [14], extended this result to give upper and lower bounds on the size of a random greedy independent set in any $d$-regular graph of girth at least $g$, which converge to the expression in (1.5) as $g \rightarrow \infty$.

The problem of finding the size of a greedy independent set in a more general random graph with given degrees was first studied in a recent preprint by Bermolen, Jonckheere and Moyal [2]. Their approach is superficially similar to ours, in that they construct the graph and the independent set simultaneously, but their analysis is significantly more complicated. (We explain the difference in more detail once we have described our process in Section 2.) Under the assumption that the 6th moment of the degree sequence is bounded, they prove that their process is approximated by the unique solution of an infinite-dimensional differential equation. The paper gives no explicit form for the solution (except in the case of a random 2regular graph, and for the Poisson distribution; in the latter case, the authors substitute a Poisson distribution for the number of empty vertices of degree $k$ and show that this satisfies their equations), and the differential equation itself involves the second moment of the degree sequence. The authors evaluate the solution numerically in several explicit instances, and extract the jamming constant.

We provide a simple system of approximating differential equations involving only the first moment of the degree sequence, and a time change where they have a simple and explicit solution. We also require only first moment conditions on the degree sequence for our result on the multigraph $G^{*}\left(n,\left(d_{i}\right)_{1}^{n}\right)$; we assume that the second moment is uniformly bounded to transfer this result to the random graph $G\left(n,\left(d_{i}\right)_{1}^{n}\right)$.

The simple greedy process for creating a random independent set is of interest for its own sake. In chemistry and physics, this process is called random sequential adsorption, and is studied, for instance, as a model of the deposition of a thin film of liquid onto a crystal. See the surveys of Evans [8] and Cadilhe, Araújo and Privman [5], and the many further references therein, for information on applications of the greedy algorithm and its variants. In statistics, the greedy process is known as simple sequential inhibition; see for instance Diggle $[6, \S 6.7]$. In these and other application areas, 
there is a "continuum" version, typically involving a greedy process for packing $d$-dimensional unit cubes into $[0, M]^{d}$ for $M$ large. In this process, unit cubes arrive in sequence, choose a location for their bottom corner uniformly at random in $[0, M-1]^{d}$, and occupy the space if no already-placed cube overlaps it. The one-dimensional version is Rényi's car-parking process [26]. See Penrose [25] for rigorous results on this car-parking process in higher dimensions. There is often also a discrete version, typically taking place on a regular lattice, where an object arrives and selects a location on the lattice uniformly at random, and then inhibits later objects from occupying neighbouring points. Bermolen, Jonckheere and Moyal [2] and Finch [10, §5.3] also list a number of other application areas, e.g., to linguistics, sociology and computer science, again with further references.

Wormald [27] also analysed a more complicated algorithm that produces, w.h.p., a larger independent set in a random $d$-regular graph on $n$ vertices. Subsequent interest in this line of research has indeed mostly been focussed on finding good bounds on the size $I\left(G_{n, d}\right)$ of a largest independent set in a random $d$-regular graph $G_{n, d}$, and in particular its expectation $\mathbb{E} I\left(G_{n, d}\right)$ : a standard argument shows that $I\left(G_{n, d}\right)$ is concentrated around its mean with fluctuations of the order at most $\sqrt{n}$. Bollobás [4] had earlier used a first moment argument to give an upper bound on $\mathbb{E} I\left(G_{n, d}\right)$ of the form $n \gamma^{+}(d)+o(n)$, where $\gamma^{+}(d)$ is a function such that

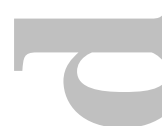

$$
\gamma^{+}(d)=\frac{2}{d}(\log d-\log \log d+1-\log 2+o(1))
$$

as $d \rightarrow \infty$ (whereas the greedy independent set has size approximately $n \log d / d$ for large $d)$. Frieze and Luczak [12] gave a lower bound on $\mathbb{E} I\left(G_{n, d}\right)$ of the form $n \gamma^{-}(d)+o(n)$, where $\gamma^{-}$has the same asymptotic behaviour as in (1.6). Bayati, Gamarnik and Tetali [1] resolved the long-standing open question of showing that there is, for each $d$, a constant $\gamma(d)$ such that $\mathbb{E} I\left(G_{n, d}\right)=n \gamma(d)+o(n)$. Recently, Ding, Sly and Sun [7] showed that, for $d$ greater than some (large) $d_{0}, I\left(G_{n, d}\right)$ has constant fluctuations about a function of the form $\alpha_{d} n+\beta_{d} \log n$, where $\alpha_{d}$ and $\beta_{d}$ are constants whose values solve some explicitly given equations. We are not aware of any work on the largest independent set in a more general random graph with given vertex degrees.

We now investigate the formulae of Theorem 1.1.

If $p_{0}+p_{1}=1$, then we find that $\tau_{\infty}=\infty$, and the formula (1.3) for the limit of $S_{\infty} / n$ evaluates to $p_{0}+\frac{1}{2} p_{1}$, which is the expected answer: indeed if a multigraph has $n_{0}$ isolated vertices, $n_{1}=n-n_{0}-o(n)$ vertices of degree 1 , and $\frac{1}{2} n_{1}+o(n)$ edges in total, then any maximal independent set has size $n_{0}+\frac{1}{2} n_{1}+o(n)$. 
We shall thus assume from now on that there is some $\ell \geq 2$ such that $p_{\ell}>0$. In this case, we see that

$$
\frac{\lambda e^{-2 \sigma}}{\sum_{k} k p_{k} e^{-k \sigma}}=\frac{\sum_{k} k p_{k} e^{-2 \sigma}}{\sum_{k} k p_{k} e^{-k \sigma}}>e^{-\sigma}
$$

for all $\sigma>0$, and hence

$$
\int_{0}^{\infty} \frac{\lambda e^{-2 \sigma}}{\sum_{k} k p_{k} e^{-k \sigma}} d \sigma>\int_{0}^{\infty} e^{-\sigma} d \sigma=1
$$

As the integrand is positive and bounded on finite intervals, this implies that there is a unique finite value $\tau_{\infty}$ satisfying (1.2).

We illustrate Theorem 1.1 in several specific cases. First, consider a random $d$-regular (multi)graph, where $p_{d}=1$. Evaluating the integral in (1.2) and setting it equal to 1 gives

$$
1=\int_{\sigma=0}^{\tau_{\infty}} e^{(d-2) \sigma} d \sigma=\frac{1}{d-2}\left(e^{(d-2) \tau_{\infty}}-1\right),
$$

for $d \geq 3$, and so $\tau_{\infty}=\frac{\log (d-1)}{d-2}$. For $d=2$ we obtain $\tau_{\infty}=1$. Now the formula in (1.3) becomes

$$
\int_{0}^{\tau_{\infty}} e^{-2 \sigma} d \sigma=\frac{1}{2}\left(1-e^{-2 \tau_{\infty}}\right)=\frac{1}{2}\left(1-\frac{1}{(d-1)^{2 /(d-2)}}\right),
$$

for $d \geq 3$, and $\frac{1}{2}\left(1-e^{-2}\right)$ for $d=2$. This indeed agrees with Wormald's formula (1.5), as well as Flory's formula [11] for $d=2$.

We can also verify that Theorem 1.1 gives the known answer in the case of the random graph $G_{n, p}$, where $p=c / n$. In this case, the vertex degrees are random, but by conditioning on the vertex degrees we can apply the results above, with the asymptotic Poisson degree distribution $p_{k}=\frac{c^{k} e^{-c}}{k !}$, for each $k \geq 0$. We may then calculate that:

$$
\lambda=\sum_{k} k p_{k}=c ; \quad \sum_{k} p_{k} e^{-k \sigma}=e^{-c} e^{c e^{-\sigma}} ; \quad \sum_{k} k p_{k} e^{-k \sigma}=c e^{-c} e^{-\sigma} e^{c e^{-\sigma}} .
$$

One may also check that the degree distribution has bounded second moment. From (1.2), we find that

$$
1=e^{c} \int_{0}^{\tau_{\infty}} e^{-\sigma} e^{-c e^{-\sigma}} d \sigma=\frac{e^{c}}{c}\left[e^{-c e^{-\tau_{\infty}}}-e^{-c}\right],
$$

and rearranging gives

$$
e^{-\tau_{\infty}}=1-\frac{\log (c+1)}{c} .
$$

We now have from (1.3) that

$$
\frac{S_{\infty}}{n} \rightarrow \int_{0}^{\tau_{\infty}} e^{-\sigma} d \sigma=1-e^{-\tau_{\infty}}=\frac{\log (c+1)}{c},
$$

which agrees with the known value, which can be found from first principles by a short calculation; see McDiarmid [23]. 
Furthermore, still considering $G_{n, p}$ with $p=c / n$, Theorem 1.2 yields

$$
\frac{S_{\infty}(k)}{n} \rightarrow \int_{0}^{\tau_{\infty}} \frac{c^{k}}{k !} e^{-(k+1) \sigma} e^{-c e^{-\sigma}} d \sigma=\frac{1}{c} \int_{c-\log (c+1)}^{c} \frac{x^{k}}{k !} e^{-x} d x .
$$

Combining (1.10) and (1.11), we see that the asymptotic degree distribution in the random greedy independent set can be described as a mixture of $\operatorname{Po}(\mu)$, with parameter $\mu$ uniformly distributed in $[c-\log (c+1), c]$.

The structure of the remainder of the paper is as follows. In Section 2, we give a full description of our process, which generates a random multigraph $G^{*}\left(n,\left(d_{i}\right)_{1}^{n}\right)$ with the given degrees simultaneously with a greedy independent set. We also specify which variables we track through the process. In Section 3, we calculate the drift of our process. In Section 4, we write down the limit differential equations, prove that the process converges to these equations, and prove Theorem 1.1. In Section 5, we show that, for the multigraph version, the conclusions of Theorem 1.1 still hold if $\lambda_{n}:=\sum_{k} k n_{k} / n$ tends to a constant $\lambda$ that may be larger than $\sum_{k} k p_{k}$; we also consider the case where $\lambda_{n}$ tends to infinity.

\section{DESCRIPTION OF PROCESS}

The key to our analysis is a process that generates the random multigraph and the random independent set in parallel. The process analysed by
Bermolen, Jonckheere and Moyal [2] is also of this nature, but our process differs in one important way, as we point out below, and our choice gives us significantly more tractable equations to solve.

Recall from the introduction that, in the configuration model, the random multigraph $G^{*}\left(n,\left(d_{i}\right)_{1}^{n}\right)$ is constructed as follows: for each $k$, and each of the $n_{k}$ vertices $i$ with degree $d_{i}=k$, we associate $k$ half-edges with vertex $i$. These half-edges are then combined into edges by a uniformly random matching. Conditioned on $G^{*}\left(n,\left(d_{i}\right)_{1}^{n}\right)$ being simple (i.e. having no loops or multiple edges), we obtain the random graph $G\left(n,\left(d_{i}\right)_{1}^{n}\right)$, uniformly distributed over all graphs on $n$ vertices with given degree sequence $\left(d_{i}\right)_{1}^{n}$. Our process will generate $G^{*}\left(n,\left(d_{i}\right)_{1}^{n}\right)$ sequentially, by revealing the pairings of the half-edges at a chosen vertex as needed. The freedom to pair the halfedges in any order allows one to study other aspects of the random graph as we generate it, in this case a greedy independent set. This theme has already been exploited many times, e.g., see $[27 ; 18 ; 19 ; 20]$.

We analyse the following continuous-time Markovian process, which generates a random multigraph on a fixed set $\mathcal{V}=\{1, \ldots, n\}$ of $n$ vertices with pre-specified degrees, so that vertex $i$ has degree $d_{i}$ for $i=1, \ldots, n$, along with an independent set $\mathcal{S}$ in the multigraph. At each time $t \geq 0$, the vertex set $\mathcal{V}$ is partitioned into three classes:

(a) a set $\mathcal{S}_{t}$ of vertices that have already been placed into the independent set, with all half-edges out of $\mathcal{S}_{t}$ paired, 
(b) a set $\mathcal{B}_{t}$ of blocked vertices, where at least one half-edge has been paired with a half-edge from $\mathcal{S}_{t}$,

(c) a set $\mathcal{E}_{t}$ of empty vertices, from which no half-edge has yet been paired.

At all times, the only paired edges are those with at least one endpoint in $\mathcal{S}_{t}$. For $j=1,2, \ldots$, we set $\mathcal{E}_{t}(j)$ to be the set of vertices in $\mathcal{E}_{t}$ of degree $j$.

Initially all vertices are empty, i.e., $\mathcal{E}_{0}=\mathcal{V}$. Each vertex $v$ has an independent exponential clock, with rate 1 . When the clock of vertex $v \in \mathcal{E}_{t}$ goes off, the vertex is placed into the independent set and all its half-edges are paired. This results in the following changes:

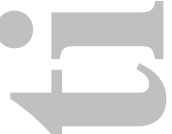

(a) $v$ is moved from $\mathcal{E}_{t}$ to $\mathcal{S}_{t}$,

(b) each half-edge incident to $v$ is paired in turn with some other uniformly randomly chosen currently unpaired half-edge,

(c) all the vertices in $\mathcal{E}_{t}$ where some half-edge has been paired with a half-edge from $v$ are moved to $\mathcal{B}_{t}$.

Note that some half-edges from $v$ may be paired with half-edges from $\mathcal{B}_{t}$, or indeed with other half-edges from $v$ : no change in the status of a vertex results from such pairings.

The clocks of vertices in $\mathcal{B}_{t}$ are ignored.

The process terminates when $\mathcal{E}_{t}$ is empty. At this point, there may still be some unpaired half-edges attached to blocked vertices: these may be paired off uniformly at random to complete the creation of the random multigraph.

The pairing generated is a uniform random pairing of all the half-edges.

The independent set generated in the random multigraph can also be described as follows: vertices have clocks that go off in a random order, and when the clock at any vertex goes off, it is placed in the independent set if possible - thus our process does generate a random greedy independent set in the random multigraph.

One particular feature of our process is that, when a vertex is moved from $\mathcal{E}_{t}$ to $\mathcal{B}_{t}$, we do not pair its half-edges. This is in contrast with the process studied by Bermolen, Jonckheere and Moyal [2], where they reveal the neighbours of blocked vertices, meaning that the degrees of empty vertices can change over time.

The variables we track in our analysis of the process are: $E_{t}(j)=\left|\mathcal{E}_{t}(j)\right|$, the number of empty vertices of degree $j$ at time $t$, for each $j \geq 0$, the total number $U_{t}$ of unpaired half-edges, and the number $S_{t}=\left|\mathcal{S}_{t}\right|$ of vertices that have so far been placed in the independent set. We claim that the vector $\left(U_{t}, E_{t}(0), E_{t}(1), \ldots, S_{t}\right)$ is Markovian. At each time $t$, there are $E_{t}(j)$ clocks associated with empty vertices of degree $j$; when the clock at one such vertex $v$ goes off, its $j$ half-edges are paired uniformly at random within the pool of $U_{t}$ available half-edges, so $U_{t}$ goes down by exactly $2 j-2 \ell$, where $\ell$ is the number of loops generated at $v$, which has a distribution that can be derived from $U_{t}$ and the degree of $v$. The distribution of the numbers of vertices of each $\mathcal{E}_{t}(k)$ that are paired with one of the half-edges out of $v$ is a 
straightforward function of the vector given. Meanwhile $S_{t}$ increases by one each time a clock at an empty vertex goes off.

\section{DRIFT}

We say that a stochastic process $X_{t}, t \geq 0$, has drift $Y_{t}$ if $X_{t}-X_{0}-\int_{0}^{t} Y_{s} d s$ is a martingale. (Or, more generally, a local martingale. In our cases, the processes are, for each $n$, bounded on finite intervals, so any local martingale is a martingale.)

Lemma 3.1. $S_{t}$ has drift

$$
\sum_{k=0}^{\infty} E_{t}(k)
$$

Proof. This is immediate, since $S_{t}$ increases by 1 each time the clock at an empty vertex goes off, and they all go off with rate 1.

Lemma 3.2. $U_{t}$ has drift

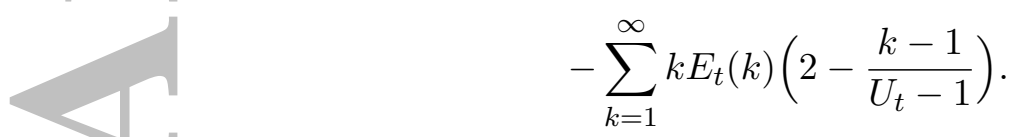

Proof. When the clock at a vertex of degree $k$ goes off, then the number of free half-edges decreases by $k+(k-2 L)=2 k-2 L$, where $L$ is the number of loops created at the vertex. We have, conditionally on $U_{t}$,

$$
\begin{aligned}
& \mathbb{E} L=\left(\begin{array}{l}
k \\
2
\end{array}\right) \frac{1}{U_{t}-1} \\
& \mathbb{E} \#\{\text { removed half-edges }\}=2 k-2 \mathbb{E} L=2 k-\frac{k(k-1)}{U_{t}-1} \\
&=k\left(2-\frac{k-1}{U_{t}-1}\right) .
\end{aligned}
$$

Now multiply by $E_{t}(k)$ and sum over $k$.

Consider two distinct vertices $v$ and $w$, of degrees $j$ and $k$, respectively, in a configuration model with $u$ half-edges. The probability that $v$ and $w$ are connected by at least one edge depends only on $j, k$ and $u$; denote it by $p_{j k}(u)$.

Lemma 3.3. $E_{t}(k)$ has drift

$$
-E_{t}(k)-\sum_{j=1}^{\infty} p_{j k}\left(U_{t}\right) E_{t}(j)\left(E_{t}(k)-\delta_{j k}\right) .
$$

Proof. When the clock at a vertex in $\mathcal{E}_{t}(j)$ (i.e., an empty vertex of degree $j$ ) goes off, that vertex is removed from the set $\mathcal{E}_{t}(j)$, which reduces $E_{t}(j)$ by 1 . Furthermore, when the clock at a vertex in $\mathcal{E}_{t}(j)$ goes off, each empty vertex of degree $k$ is joined to it with probability $p_{j k}\left(U_{t}\right)$ (since we may ignore the 
half-edges already paired). Hence the expected total decrease of $E_{t}(k)$ when a vertex in $\mathcal{E}_{t}(j)$ goes off is $p_{j k}\left(U_{t}\right) E_{t}(k)$ when $j \neq k$ and $1+p_{j k}\left(U_{t}\right)\left(E_{t}(k)-1\right)$ when $j=k$. Now multiply by $E_{t}(j)$ and sum over $j$.

Let $(k)_{m}=k(k-1) \cdots(k-m+1)$ denote the falling factorials.

Lemma 3.4. We have, with the sums really finite and extending only to $j \wedge k$,

$$
\begin{aligned}
p_{j k}(u) & =\sum_{m=1}^{\infty} \frac{(-1)^{m-1}}{m !} \frac{(j)_{m}(k)_{m}}{(u-1)(u-3) \cdots(u-2 m+1)} \\
& =\sum_{m=1}^{\infty} \frac{(-1)^{m-1}}{m !} \frac{(j)_{m}(k)_{m}}{2^{m}((u-1) / 2)_{m}} .
\end{aligned}
$$

Furthermore,

$$
\frac{j k}{u-1} \geq p_{j k}(u) \geq \frac{j k}{u-1}-\frac{j(j-1) k(k-1)}{2(u-1)(u-3)} .
$$

Proof. The formula (3.6) is obtained by the inclusion-exclusion principle applied to the variable $X$ defined as the number of edges between the two given vertices $v$ and $w$; or equivalently, applied to the family of events that a given pair of half-edges at $v$ and $w$ are joined. We have, for any $m \geq 0$,

$$
\mathbb{E}(X)_{m}=(j)_{m}(k)_{m} \frac{1}{(u-1)(u-3) \cdots(u-2 m+1)},
$$

since the left-hand side is the expected number of sequences of pairs $\left(x_{1}, y_{1}\right)$, $\ldots,\left(x_{m}, y_{m}\right)$ where the $x_{i}$ are distinct half-edges at $v$ and the $y_{i}$ are distinct half-edges at $w$, and $x_{i}$ is joined to $y_{i}$ in the pairing; the $x_{i}$ and $y_{i}$ can be chosen in $(j)_{m}(k)_{m}$ ways, and the final factor in (3.8) is the probability that any given sequences $\left(x_{i}\right)$ and $\left(y_{i}\right)$ are paired. Then, (3.6) is given by the standard formula (see Feller [9, IV.1], in an equivalent form)

$$
\mathbb{P}(X \geq 1)=\sum_{m=1}^{\infty}(-1)^{m-1} \mathbb{E}\left(\begin{array}{l}
X \\
m
\end{array}\right)=\sum_{m=1}^{\infty} \frac{(-1)^{m-1}}{m !} \mathbb{E}(X)_{m}
$$

Similarly, the inequalities (3.7) are instances of the Bonferroni inequalities (see Feller [9, IV.5(c)]).

Remark 3.5. The sum in (3.6) is (a minor modification of) a hypergeometric sum; in terms of the hypergeometric function ${ }_{2} F_{1}$, we have

$$
p_{j k}(u)=1-{ }_{2} F_{1}\left(-j,-k ; \frac{1-u}{2} ; \frac{1}{2}\right) .
$$

\section{Analysis: Proof of Theorem 1.1}

First, recall that $\sum_{k} k n_{k} / n \rightarrow \lambda$. Since it suffices to consider large $n$, we may without loss of generality assume that

$$
\sum_{k} k n_{k} \leq 2 \lambda n
$$


Next, by Lemmas 3.2 and 3.3, we can write

$$
U_{t}=U_{0}-\int_{0}^{t} \sum_{k=1}^{\infty} k E_{s}(k)\left(2-\frac{k-1}{U_{s}-1}\right) d s+M_{t},
$$

and, for each $k \in \mathbb{Z}^{+}$,

$$
E_{t}(k)=E_{0}(k)-\int_{0}^{t} E_{s}(k) d s-\int_{0}^{t} \sum_{j=1}^{\infty} p_{j k}\left(U_{s}\right) E_{s}(j)\left(E_{s}(k)-\delta_{j k}\right) d s+M_{t}(k),
$$

where $M_{t}$ and each $M_{t}(k)$ is a martingale.

Dividing by $n$,

$$
\frac{U_{t}}{n}=\frac{U_{0}}{n}-\int_{0}^{t} \sum_{k=1}^{\infty} \frac{k E_{s}(k)}{n}\left(2-\frac{k-1}{U_{s}-1}\right) d s+\frac{M_{t}}{n},
$$

and, for each $k$,

$$
\frac{E_{t}(k)}{n}=\frac{E_{0}(k)}{n}-\int_{0}^{t} \frac{E_{s}(k)}{n} d s-\int_{0}^{t} \sum_{j=1}^{\infty} p_{j k}\left(U_{s}\right) \frac{E_{s}(j)}{n}\left(E_{s}(k)-\delta_{j k}\right) d s+\frac{M_{t}(k)}{n} .
$$

The martingale $M_{t}$ has by (4.2) locally finite variation, and hence its quadratic variation $[M]_{t}$ is given by

$$
[M]_{t}=\sum_{0 \leq s \leq t}\left(\Delta M_{s}\right)^{2}=\sum_{0 \leq s \leq t}\left(\Delta U_{s}\right)^{2} \leq \sum_{s \geq 0}\left(\Delta U_{s}\right)^{2} \leq \sum_{j}(2 j)^{2} n_{j} .
$$

Now, since $\sum_{k=1}^{\infty} k n_{k} / n$ is uniformly summable, for every $\varepsilon>0$, there exists $J$ such that $n^{-1} \sum_{j>J} j n_{j} \leq \varepsilon$ for all large enough $n$. So, for $\varepsilon>0$, for $n$ large enough, we have

$$
\sum_{j}(2 j)^{2} n_{j}=\sum_{j \leq J}(2 j)^{2} n_{j}+\sum_{j>J}(2 j)^{2} n_{j} \leq 4 J^{2} n+4 \varepsilon n^{2} \leq 5 \varepsilon n^{2} .
$$

Since $\varepsilon$ can be taken arbitrarily small, it follows that $[M]_{t}=o\left(n^{2}\right)$. (In fact, $[M]_{t}=O(n)$ if the second moment of the degree distribution is uniformly bounded.) Likewise, for each $k$,

$$
[M(k)]_{t} \leq \sum_{s \geq 0}\left(\Delta E_{s}(k)\right)^{2} \leq \sum_{j}(j+1)^{2} n_{j}=o\left(n^{2}\right),
$$

with the bound being $O(n)$ if the second moment of the degree distribution is uniformly bounded. Doob's inequality then gives $\sup _{t \geq 0}\left|M_{t}\right|=o_{\mathrm{p}}(n)$ and, for each $k, \sup _{t>0}\left|M_{t}(k)\right|=o_{\mathrm{p}}(n)$.

Now the integrand in (4.4) is bounded above by $2 \sum_{k=1}^{\infty} k n_{k} / n$, which is less than $4 \lambda$ by (4.1). Also, the integrand in the first integral in (4.5) is bounded above by 1 , and the integrand in the second integral is bounded above by $4 \lambda$, since $p_{j k}\left(U_{t}\right) \leq j k /\left(U_{t}-1\right) \leq 2 j k / U_{t}$ and $\sum_{j=1}^{\infty} j E_{s}(j) \leq U_{s}$ (there are at least as many free half-edges, as there are free half-edges at empty vertices), 
It follows that $\left(U_{t}-M_{t}\right) / n, n \geq 1$, is a uniformly Lipschitz family, and it is also uniformly bounded on each finite interval $\left[0, t_{0}\right]$. Likewise, for each $k,\left(E_{t}(k)-M_{t}(k)\right) / n, n \geq 1$, is a uniformly Lipschitz family, and it is also uniformly bounded on each finite interval $\left[0, t_{0}\right]$. Thus, the ArzelaAscoli theorem implies that each of the above families is tight in $C\left[0, t_{0}\right]$ for any $t_{0}>0[21$, Theorems A2.1 and 16.5], and so also in $C[0, \infty)[21$, Theorem 16.6]. This then implies that, for each of the above processes, there exists a subsequence along which the process converges in distribution in $C[0, \infty)$. Since there are countably many processes, we can find a common subsequence where

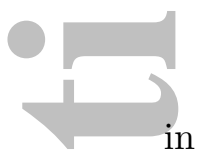

$$
\frac{U_{t}-M_{t}}{n} \rightarrow u_{t} \quad \text { and each } \quad \frac{E_{t}(k)-M_{t}(k)}{n} \rightarrow e_{t}(k)
$$

in distribution in $C[0, \infty)$, for some random continuous functions $u_{t}$ and $e_{t}(k)$. By the Skorokhod coupling lemma [21, Theorem 4.30] we may assume that the limits in (4.8) hold almost surely (in $C[0, \infty)$, i.e., uniformly on compact sets), and also that $\sup _{t \geq 0}\left|M_{t}\right| / n \stackrel{\text { a.s. }}{\longrightarrow} 0$ and $\sup _{t \geq 0}\left|M_{t}(k)\right| / n \stackrel{\text { a.s. }}{\longrightarrow}$ 0 . Hence, along the subsequence, a.s.

$$
\frac{U_{t}}{n} \rightarrow u_{t} \quad \text { and each } \quad \frac{E_{t}(k)}{n} \rightarrow e_{t}(k)
$$

uniformly on compact sets, with continuous limits $u_{t}$ and $e_{t}(k), k=0,1,2, \ldots$ Since $U_{t} \geq 0$ and $E_{t}(k) \geq 0$, we must have $u_{t} \geq 0$ and $e_{t}(k) \geq 0$ for all $t$ and $k$. Clearly, $u_{0}=\lambda$ and $e_{0}(k)=p_{k}$.

The next step is to show that the random functions $u_{t}$ and $e_{t}(k)$ satisfy the system of equations (4.28)-(4.29) below. Note that we have, by (4.4), for $0 \leq r<t<\infty$, as $\sum_{k} k E_{s}(k) \leq U_{s}$,

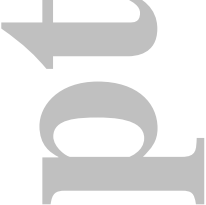

$$
\begin{aligned}
\frac{U_{t}}{n}-\frac{U_{r}}{n} & \geq-2 \int_{r}^{t} \sum_{k=1}^{\infty} \frac{k E_{s}(k)}{n} d s+\frac{M_{t}}{n}-\frac{M_{r}}{n} \\
& \geq-2 \int_{r}^{t} \frac{U_{s}}{n} d s+\frac{M_{t}}{n}-\frac{M_{r}}{n} .
\end{aligned}
$$

This, and the fact that $U_{t}$ is non-increasing, then implies that

$$
0 \geq u_{t}-u_{r} \geq-2 \int_{r}^{t} u_{s} d s .
$$

This (or (4.8) and the argument preceding it) shows that $u_{t}$ is a Lipschitz function. Thus $u_{t}$ is differentiable a.e., and (4.11) implies $\frac{d}{d t} u_{t} \geq-2 u_{t}$ and thus $\frac{d}{d t}\left(e^{2 t} u_{t}\right) \geq 0$ a.e. A Lipschitz function is absolutely continuous and thus the integral of its derivative; hence it follows that $e^{2 t} u_{t}$ is non-decreasing and hence that $u_{t} \geq e^{-2 t} u_{0}=\lambda e^{-2 t}>0$ for all $t$.

In particular, (4.9) implies that along the subsequence, a.s. $U_{t} \rightarrow \infty$ for every fixed $t$. 
Thus, a.s., along the subsequence, $(k-1) /\left(U_{s}-1\right) \rightarrow 0$ for every fixed $k$ and $s<\infty$, and so, using (4.9),

$$
a_{s}(k):=\frac{k E_{s}(k)}{n}\left(2-\frac{k-1}{U_{s}-1}\right) \rightarrow 2 k e_{s}(k) .
$$

Furthermore,

$$
a_{s}(k) \leq \frac{2 k E_{0}(k)}{n}=\frac{2 k n_{k}}{n} .
$$

By assumption, $k n_{k} / n$ is uniformly summable, and by (4.13) so is $a_{s}(k)$. Hence (4.12) yields

$$
A_{s}:=\sum_{k=1}^{\infty} a_{s}(k) \rightarrow 2 \sum_{k=1}^{\infty} k e_{s}(k) .
$$

Furthermore, by (4.13) and (4.1), for all $s \geq 0$,

$$
A_{s} \leq \frac{2 \sum_{k=1}^{\infty} k n_{k}}{n} \leq 4 \lambda \text {. }
$$

Hence, by dominated convergence, for any $t<\infty$, a.s.

$$
\int_{0}^{t} A_{s} d s \rightarrow 2 \int_{0}^{t} \sum_{k=1}^{\infty} k e_{s}(k) d s .
$$

Note that the integral in (4.4) is $\int_{0}^{t} A_{s} d s$. Hence, taking the limit in (4.4) as $n \rightarrow \infty$ along the subsequence yields, using (4.9) and $M_{t} / n \rightarrow 0$ a.s.,

$$
u_{t}=\lambda-2 \int_{0}^{t} \sum_{k=1}^{\infty} k e_{s}(k) d s .
$$

Now, fix $k \in \mathbb{Z}^{+}$, and, for each $j \in \mathbb{Z}^{+}$, let

$$
b_{s}(j)=p_{j k}\left(U_{s}\right) \frac{E_{s}(j)}{n}\left(E_{s}(k)-\delta_{j k}\right) .
$$

By Lemma 3.4 and (4.9), a.s., for each fixed $s \geq 0$ and $j \in \mathbb{Z}^{+}$,

$$
\begin{gathered}
n p_{j k}\left(U_{s}\right)=\frac{n j k}{U_{s}-1}\left(1+O\left(\frac{j k}{U_{s}-1}\right)\right) \rightarrow \frac{j k}{u_{s}} \\
\frac{E_{s}(j)}{n} \frac{E_{s}(k)-\delta_{j k}}{n} \rightarrow e_{s}(j) e_{s}(k) .
\end{gathered}
$$

Hence, a.s., for each fixed $s \geq 0$ and $j \in \mathbb{Z}^{+}$,

$$
b_{s}(j) \rightarrow \frac{j k}{u_{s}} e_{s}(j) e_{s}(k) .
$$

Furthermore, if $U_{s}=0$ then $E_{s}(j)=0$ and $b_{s}(j)=0$, and if $U_{s}>0$ then

$$
0 \leq b_{s}(j) \leq \frac{j k}{U_{s}-1} \frac{E_{s}(j) E_{s}(k)}{n} \leq 2 \frac{k E_{s}(k)}{U_{s}} \frac{j E_{s}(j)}{n} \leq 2 \frac{j n_{j}}{n} .
$$


Thus $b_{s}(j)$ is uniformly summable and (4.21) yields

$$
\sum_{j} b_{s}(j) \rightarrow k e_{s}(k) \sum_{j} \frac{j e_{s}(j)}{u_{s}} .
$$

Moreover, by (4.22) and (4.1), for every $s$,

$$
\sum_{j} b_{s}(j) \leq \frac{2 \sum_{j} j n_{j}}{n} \leq 4 \lambda .
$$

Consequently, by dominated convergence, for any $t<\infty$,

$$
\int_{0}^{t} \sum_{j} b_{s}(j) d s \rightarrow \int_{0}^{t} k e_{s}(k) \sum_{j} \frac{j e_{s}(j)}{u_{s}} d s \quad \text { a.s. }
$$

The second integral in (4.5) is $\int_{0}^{t} \sum_{j} b_{s}(j) d s$, and for the first integral we have directly by (4.9) and dominated convergence

$$
\int_{0}^{t} \frac{E_{s}(k)}{n} d s \rightarrow \int_{0}^{t} e_{s}(k) d s \quad \text { a.s. }
$$

Since also $M_{t}(k) / n \rightarrow 0$ a.s., we thus see from (4.5) that

$$
e_{t}(k)=p_{k}-\int_{0}^{t} e_{s}(k) d s-\int_{0}^{t} k e_{s}(k) \frac{\sum_{j=1}^{\infty} j e_{s}(j)}{u_{t}} d s .
$$

In other words, we have shown that the subsequential a.s. limit $\left(u_{t}, e_{t}(k)\right.$ : $k=0,1, \ldots)$ of $\left(U_{t} / n, E_{t}(k) / n: k=0,1, \ldots\right)$ a.s. must satisfy the following system of equations:

$$
\begin{aligned}
u_{t} & =\lambda-2 \int_{0}^{t} \sum_{k=1}^{\infty} k e_{s}(k) d s \\
e_{t}(k) & =p_{k}-\int_{0}^{t} e_{s}(k) d s-\int_{0}^{t} k e_{s}(k) \frac{\sum_{j=1}^{\infty} j e_{s}(j)}{u_{s}} d s, \quad k \in \mathbb{Z}^{+} .
\end{aligned}
$$

Below, we will show that the equations (4.28)-(4.29) have a unique solution $\left(u_{t}, e_{t}(k): k=0,1, \ldots\right)$. This means that $u_{t}$ and $e_{t}(k)$, which a priori are random, in fact are deterministic. Moreover, since the limits $u_{t}$ and $e_{t}(k)$ are continuous, convergence uniformly on compact sets in (4.9) is equivalent to convergence in the Skorokhod space $D[0, \infty)$. Consequently, the argument above shows that each subsequence of $\left(U_{t} / n, E_{t}(k) / n: k=0,1, \ldots\right)$ has a subsequence which converges in distribution in the Skorokhod topology, and that each convergent subsequence converges to the same $\left(u_{t}, e_{t}(k): k=\right.$ $0,1, \ldots)$. This implies that the whole sequence $\left(U_{t} / n, E_{t}(k) / n: k=0,1, \ldots\right)$ must in fact converge to $\left(u_{t}, e_{t}(k): k=0,1, \ldots\right)$ in distribution in the Skorokhod topology. Since the limit is deterministic, the convergence holds in probability.

We proceed to solve (4.28)-(4.29). (This is a system of equations of deterministic functions; there is no randomness in this part of the proof.) We use also the facts $e_{t}(k) \geq 0$ and $u_{t}>0$ established above. 
First of all, each $e_{s}(k)$ is continuous. Furthermore, e.g. by $(4.29), e_{s}(k)$ is bounded by $e_{0}(k)=p_{k}$ with $\sum_{k} k p_{k}<\infty$. Hence the sum $\sum_{k} k e_{s}(k)$ converges uniformly and thus this sum is continuous. Since also $u_{s}$ is continuous and $u_{s}>0$, the integrands in the integrals in (4.28)-(4.29) are continuous. Consequently, the functions $u_{t}$ and $e_{t}(k)$ are continuously differentiable and the integral equations (4.28)-(4.29) can be written as a system of differential equations

$$
\begin{aligned}
\frac{d u_{t}}{d t} & =-2 \sum_{k=1}^{\infty} k e_{t}(k) \\
\frac{d e_{t}(k)}{d t} & =-e_{t}(k)-k e_{t}(k) \frac{\sum_{j=1}^{\infty} j e_{t}(j)}{u_{t}}, \quad k \in \mathbb{Z}^{+} .
\end{aligned}
$$

with the initial conditions $u_{0}=\lambda$ and $e_{0}(k)=p_{k}$.

Note that the system (4.30)-(4.31) is infinite, and it is not a priori obvious that it has a solution, or that the solution is unique. The system is not obviously Lipschitz with respect to any of the usual norms on sequence spaces. Fortunately, it is possible to decouple the system via a change of variables and a time-change, leaving us with an explicit solution in terms of the new variables.

We make the change of variables

$$
h_{t}(j)=e^{t} e_{t}(j)
$$

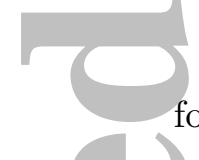

for each $j$. Note that $\frac{d h_{t}(j)}{d t}=e^{t} \frac{d e_{t}(j)}{d t}+e^{t} e_{t}(j)$, so, from (4.31) we obtain

$$
\frac{d h_{t}(k)}{d t}=-k h_{t}(k) \frac{\sum_{j} j e_{t}(j)}{u_{t}} .
$$

Now we rescale time by introducing a new time variable $\tau=\tau_{t}$ such that

$$
\frac{d \tau}{d t}=\frac{\sum_{j} j e_{t}(j)}{u_{t}}
$$

with $\tau_{0}=0$. This is well defined as $u_{t}>0$ for all $t$. Note that $\sum_{j} j e_{t}(j) \leq$ $\sum_{j} j p_{j}=\lambda$; hence (4.31) implies that for any $T>0$ and $t \in[0, T]$,

$$
\frac{d e_{t}(k)}{d t} \geq-\left(1+\frac{\lambda}{u_{T}} k\right) e_{t}(k)
$$

and thus $e_{t}(k) \geq p_{k} \exp \left\{-\left(1+k \lambda / u_{T}\right) t\right\}$ for $t \in[0, T]$. In particular, if $p_{k}>$ 0 , then $e_{t}(k)>0$ for all $t \geq 0$. Hence, $\sum_{k=1}^{\infty} k e_{t}(k)>0$ for all $t$, and thus $d \tau / d t>0$ for all $t$, so $\tau$ is a strictly increasing function of $t$. Consequently, the mapping $t \mapsto \tau_{t}$ is a bijection of $[0, \infty)$ onto $\left[0, \tau_{\infty}\right)$ for some $\tau_{\infty} \in(0, \infty]$; furthermore, this map and its inverse are both continuously differentiable.

We can thus regard the functions $u_{t}, e_{t}(j)$ and $h_{t}(j)$ as functions of $\tau \in$ $\left[0, \tau_{\infty}\right)$; we denote these simply by $u_{\tau}, e_{\tau}(j)$ and $h_{\tau}(j)$. With this convention, 
we obtain from $(4.30),(4.33)$ and (4.34) that

$$
\begin{aligned}
\frac{d u_{\tau}}{d \tau} & =\frac{d u}{d t} \frac{d t}{d \tau}=-2 u_{\tau}, \\
\frac{d h_{\tau}(j)}{d \tau} & =\frac{d h(j)}{d t} \frac{d t}{d \tau}=-j h_{\tau}(j), \quad j \in \mathbb{Z}^{+},
\end{aligned}
$$

subject to initial conditions $u_{0}=\lambda$ and $h_{0}(j)=p_{j}$. The system (4.36)(4.37) has the obvious unique solution

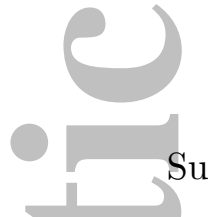

$$
\begin{aligned}
u_{\tau} & =\lambda e^{-2 \tau} \\
h_{\tau}(j) & =p_{j} e^{-j \tau}, \quad j \in \mathbb{Z}^{+} .
\end{aligned}
$$

Substituting (4.32) and (4.38)-(4.39) into (4.34) yields

$$
\frac{d \tau}{d t}=e^{-t} \frac{\sum_{k} k p_{k} e^{-k \tau}}{\lambda e^{-2 \tau}}
$$

and separating the variables and using the boundary conditions gives

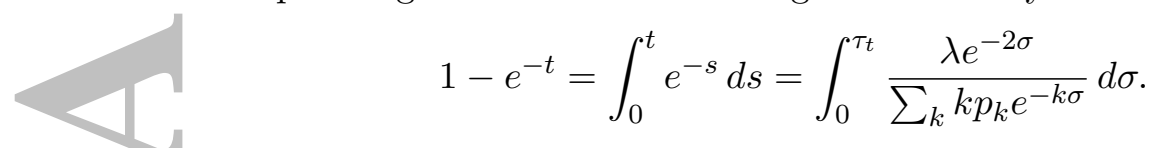

Since the integrand is positive, this determines $\tau_{t}$ uniquely for every $t \in$ $[0, \infty)$, and thus $u_{t}, h_{t}(j)$ and $e_{t}(j)$ are determined by (4.38)-(4.39) and (4.32). This completes the proof that the equations (4.28)-(4.29) have a unique solution, at least assuming $e_{t}(k) \geq 0$ and $u_{t}>0$, which any subsequential limit of our process must satisfy, as shown above.

Furthermore, letting $t \rightarrow \infty$ in (4.41),

$$
\int_{0}^{\tau_{\infty}} \frac{\lambda e^{-2 \sigma}}{\sum_{k} k p_{k} e^{-k \sigma}} d \sigma=1
$$

so $\tau_{\infty}$ is the value given by (1.2). As we saw already in Section 1 , this determines $\tau_{\infty}$ uniquely.

It is time to consider the variable that we really are interested in, viz. $S_{t}$.

By Lemma 3.1 (and $S_{0}=0$ ), we have, in analogy with (4.2)-(4.3),

$$
S_{t}=\int_{0}^{t} \sum_{k=0}^{\infty} E_{s}(k) d s+\tilde{M}_{t},
$$

for some martingale $\tilde{M}_{t}$. All jumps $\Delta S_{t}$ are equal to 1 , and it follows as in (4.6) and (4.7) that

$$
[\tilde{M}]_{t} \leq \sum_{s \geq 0}\left(\Delta S_{s}\right)^{2} \leq n
$$

and Doob's inequality gives $\sup _{t \geq 0}\left|\tilde{M}_{t}\right|=o_{\mathrm{p}}(n)$. Furthermore, we have shown that (4.9) holds (for the full sequence) with convergence in probability in $D[0, \infty)$, and again we may, by the Skorokhod coupling lemma, assume that the convergence holds a.s. in $D[0, \infty)$, and thus uniformly on compact 
intervals. Since $E_{s}(k) / n \leq n_{k} / n$ and $\sum_{k=0}^{\infty} n_{k} / n$ is uniformly summable (since $\sum_{k} k n_{k} / n$ is), it then follows that

$$
\sum_{k=0}^{\infty} \frac{E_{s}(k)}{n} \rightarrow \sum_{k=0}^{\infty} e_{s}(k) \quad \text { a.s. }
$$

for every $s$. Furthermore, trivially $\sum_{k=0}^{\infty} E_{s}(k) / n \leq 1$; hence (4.43) and (4.45) yield, by dominated convergence, a.s.

$$
\frac{S_{t}}{n} \rightarrow s_{t}:=\int_{0}^{t} \sum_{k=0}^{\infty} e_{s}(k) d s
$$

for any $t<\infty$. We want to extend this to $t=\infty$.

Since $S_{t} \leq n$, we see from (4.46) that $s_{t} \leq 1$ for every $t<\infty$; hence

$$
s_{\infty}=\lim _{t \rightarrow \infty} s_{t}=\int_{0}^{\infty} \sum_{k=0}^{\infty} e_{t}(k) d t
$$

is finite. This can also be seen directly since $\sum_{k=0}^{\infty} e_{t}(k) \leq e^{-t}$ by (4.32) and (4.39). In particular, $\sum_{k=0}^{\infty} e_{t}(k) \rightarrow 0$ as $t \rightarrow \infty$.

Let $\varepsilon>0$. Then there exists $T=T(\varepsilon)<\infty$ such that $s_{\infty}-s_{T}<\varepsilon$ and $\sum_{k=0}^{\infty} e_{T}(k)<\varepsilon$. Now,

$$
\left|\frac{S_{\infty}}{n}-s_{\infty}\right| \leq\left|\frac{S_{\infty}-S_{T}}{n}\right|+\left|\frac{S_{T}}{n}-s_{T}\right|+\left|s_{T}-s_{\infty}\right| .
$$

The last term in (4.48) is less than $\varepsilon$ by our choice of $T$. Furthermore, by (4.46), the term $\left|S_{T} / n-s_{T}\right|$ tends to 0 a.s., and thus in probability. Hence, $\left|S_{T} / n-s_{T}\right| \leq \varepsilon$ w.h.p. (meaning, as before, with probability tending to 1 as $n \rightarrow \infty)$. Moreover, for any $t, 0 \leq S_{\infty}-S_{t} \leq \sum_{k=0}^{\infty} E_{t}(k)$, and by (4.45),

$$
\sum_{k=0}^{\infty} \frac{E_{T}(k)}{n} \rightarrow \sum_{k=0}^{\infty} e_{T}(k)<\varepsilon
$$

a.s. and thus in probability. Hence also $\left(S_{\infty}-S_{T}\right) / n<\varepsilon$ w.h.p. Consequently, (4.48) shows that $\left|S_{\infty} / n-s_{\infty}\right|<3 \varepsilon$ w.h.p. Since $\varepsilon>0$ is arbitrary, we have shown that

$$
\frac{S_{\infty}}{n} \rightarrow s_{\infty}
$$

in probability, and it remains only to show that the jamming constant $s_{\infty}$ equals the constant in (1.3).

We use (4.47) and make the same change of variables $t \mapsto \tau$ as before.

Thus, using (4.34),

$$
\begin{aligned}
s_{\infty} & =\int_{0}^{\infty} \sum_{k} e_{t}(k) d t=\int_{0}^{\tau_{\infty}} \sum_{k} e_{\tau}(k) \frac{u_{\tau}}{\sum_{k} k e_{\tau}(k)} d \tau \\
& =\int_{0}^{\tau_{\infty}} \frac{u_{\tau} \sum_{k} h_{\tau}(k)}{\sum_{k} k h_{\tau}(k)} d \tau .
\end{aligned}
$$


Substituting our explicit expressions (4.38) and (4.39) for $u_{\tau}$ and the $h_{\tau}(k)$ yields

$$
s_{\infty}=\lambda \int_{0}^{\tau_{\infty}} e^{-2 \tau} \frac{\sum_{k} p_{k} e^{-k \tau}}{\sum_{k} k p_{k} e^{-k \tau}} d \tau
$$

as in Theorem 1.1.

This completes the proof of Theorem 1.1. Theorem 1.2 follows by simple modifications of the final part of the proof above: The drift of $S_{t}(k)$ is $E_{t}(k)$ and it follows as in (4.46) that

$$
\frac{S_{t}(k)}{n} \rightarrow s_{t}(k):=\int_{0}^{t} e_{s}(k) d s
$$

for any $t<\infty$. Arguing as in (4.48)-(4.50), we see that (4.53) holds for $t=\infty$ too, and (1.4) follows in analogy with (4.51) and (4.52).

\section{Without UNIFORM INTEGRABILITY}

In this section, we discuss what happens when we relax the condition of uniform integrability of the degree distribution, i.e., uniform summability of $k n_{k} / n$. This is relevant only for the multigraph case, since for the graph case we have to assume $\sum_{k} k^{2} n_{k}=O(n)$ for our proof, and then $k n_{k} / n$ is always uniformly summable. Recall our convention that looped vertices are eligible for the independent set: for part of the range of heavy-tailed distributions we consider here, many vertices of larger degree will have loops, so this choice has a significant impact on the behaviour, see Example 5.5.

We still assume that the number $n_{k}$ of vertices of degree $k$ satisfies $n_{k} / n \rightarrow$ $p_{k}$ for $k=0,1, \ldots$, where $p_{k} \geq 0$, and to avoid trivialities we assume $p_{0}<$ 1 , but we allow $\sum_{k=0}^{\infty} p_{k}<1$. We denote the average degree by $\lambda_{n}:=$ $\sum_{k=1}^{\infty} k n_{k} / n$ and assume that $\lambda_{n}$ converges to some $\lambda \leq \infty$.

We first treat the case when $\lambda<\infty$. Then the degree distributions are tight, so their limit $\left(p_{k}\right)_{0}^{\infty}$ is a probability distribution; furthermore, by Fatou's lemma, the mean $\mu=\sum_{k=1}^{\infty} k p_{k}$ of this limiting degree distribution satisfies $\mu \leq \lambda<\infty$, so the probability distribution $\left(p_{k}\right)_{0}^{\infty}$ has a finite and non-zero mean $\mu$. (It is possible that $\mu<\lambda$; see for instance Example 5.5 below.)

The proof in Section 4 uses uniform summability of $k n_{k} / n$ to show that the quadratic variation of martingales $\left(M_{t}\right)$ and $\left(M_{t}(k)\right)$ is $o\left(n^{2}\right)$ in (4.6) and (4.7); furthermore, we use it to justify taking limits under the summation sign in (4.14), (4.23) and (4.45). We give in this section an alternative (but longer) argument for the multigraph case that does not use the uniform summability.

We let $c_{1}, C_{1}, \ldots$ denote positive constants that may depend on the collection $\left\{n_{k}(n)\right\}_{n, k \geq 1}$ and $\left(p_{k}\right)_{0}^{\infty}$, but not on $n$. We replace (4.1) by $\sum_{k} k n_{k} \leq$ $C_{1} n$.

Lemma 5.1. There exists $c_{1}>0$ such that for every $t \in[0,1]$,

$$
\mathbb{E} E_{t}(k) \leq 2 n_{k} e^{-c_{1} k t}, \quad k \in \mathbb{Z}^{+} .
$$


Proof. We may assume that $n$ is large. (For any fixed $n, n_{k}>0$ only for finitely many $k$; furthermore, $\mathbb{E} E_{t}(k) \leq e^{-t} n_{k}$ and (5.1) follows if $c_{1}$ is small enough.)

Assume $0<t \leq 1$. Fix some $\ell \geq 1$ with $p_{\ell}>0$, and consider only $n$ that are so large that $\frac{1}{2} p_{\ell}<n_{\ell} / n<2 p_{\ell}$. Let $\delta:=p_{\ell} / 9$, and suppose also that $\lceil\delta n\rceil \leq n p_{\ell} / 8$. Define the stopping time $\tau_{t}$ as the time that we create the $\lceil\delta t n\rceil$-th edge (by pairing a half-edge with another). Even if there are several edges created at the same time, we regard them as created one by one, separated by infinitesimal time intervals, and we stop when exactly the right number is created. Until we stop, at most $2\lceil\delta t n\rceil \leq 2\lceil\delta n\rceil$ vertices have gone off or become blocked; in particular, at each time before we stop there are at least $n_{\ell}-2\lceil\delta n\rceil \geq \frac{1}{4} p_{\ell} n$ empty vertices of degree $\ell$. Each time one of these empty vertices goes off, at least one edge is created. (In fact, at least $\lceil(\ell+1) / 2\rceil$ edges. $)$ Hence, until we stop, the times between the creations of the edges can be dominated by i.i.d. exponential variables with mean $1 /\left(c_{2} n\right)$ (with $c_{2}=p_{\ell} / 4>\delta$ ), and thus the creation of edges dominates a Poisson process with intensity $c_{2} n$; consequently, by a Chernoff estimate,

$$
\mathbb{P}\left(\tau_{t}>t\right) \leq \mathbb{P}\left(\operatorname{Po}\left(c_{2} n t\right)<\delta t n\right) \leq e^{-c_{3} t n} .
$$

Now consider a vertex $v$ of degree $k$. As long as $v$ is empty, it has probability $k /\left(U_{t}-1\right) \geq k /\left(C_{1} n\right)$ of being blocked at each pairing of a half-edge. Hence,

and thus

$$
\mathbb{P}\left(v \text { is empty at } \tau_{t}\right) \leq\left(1-\frac{k}{C_{1} n}\right)^{\delta t n} \leq e^{-c_{4} k t},
$$

$$
\mathbb{E} E_{\tau_{t}}(k) \leq e^{-c_{4} k t} n_{k}
$$

Consequently, using (5.2) and (5.4),

$$
\mathbb{E} E_{t}(k) \leq e^{-c_{4} k t} n_{k}+\mathbb{P}\left(\tau_{t}>t\right) n_{k} \leq\left(e^{-c_{4} k t}+e^{-c_{3} n t}\right) n_{k} .
$$

Since $n_{k}>0$ only if $k \leq C_{1} n$, the result follows.

Lemma 5.2. Let $E^{*}(k)$ be the number of vertices of degree $k$ that are empty when they go off. Then

$$
\mathbb{E} E^{*}(k) \leq C_{2} \frac{n_{k}}{k} .
$$

Proof. The expected number of empty vertices of degree $k$ that go off at a time in $[0,1]$ is, using Lemma 5.1,

$$
\int_{0}^{1} \mathbb{E} E_{k}(t) d t \leq 2 n_{k} \int_{0}^{1} e^{-c_{1} k t} d t \leq C_{3} \frac{n_{k}}{k} .
$$

Furthermore, also by Lemma 5.1, the expected number of empty vertices of degree $k$ that go off after 1 is at most

$$
\mathbb{E} E_{1}(k) \leq 2 n_{k} e^{-c_{1} k} \leq C_{4} \frac{n_{k}}{k} .
$$


Lemma 5.3. For the martingales $M$ and $M(k)$ in (4.6) and (4.7),

$$
\mathbb{E}[M]_{\infty}=O(n), \quad \mathbb{E}[M(k)]_{\infty}=O(n) .
$$

Proof. Since $M$ jumps by at most $2 j$ when an empty vertex of degree $j$ goes off, and $M$ otherwise is continuous, we have, cf. (4.6), $[M]_{\infty} \leq \sum_{j}(2 j)^{2} E^{*}(j)$. Thus Lemma 5.2 implies

$$
\mathbb{E}[M]_{\infty} \leq \sum_{j=1}^{\infty}(2 j)^{2} \mathbb{E} E^{*}(j) \leq 4 C_{2} \sum_{j=1}^{\infty} j n_{j} \leq C_{5} n .
$$

Similarly, cf. (4.7), $[M(k)]_{\infty} \leq \sum_{j=0}^{\infty}(j+1)^{2} E^{*}(j)$ and $\mathbb{E}[M(k)]_{\infty} \leq C_{6} n$ follows.

This gives the estimates we need for the martingales.

Furthermore, Lemma 5.1 implies that for any fixed integer $m \geq 1$ and $t>0, \mathbb{E} \sum_{k} k^{m} E_{t}(k) / n \leq 2 \sum_{k} k^{m} e^{-c_{1} k(1 \wedge t)}=O(1)$. Hence the random variables $\sum_{k} k^{m} E_{t}(k) / n$ are tight, so, when taking subsequences in the proof, we may also assume that $\sum_{k} k^{m} E_{t}(k) / n$ converges in distribution, for any fixed $m$ (or all $m$ ) and, say, any rational $t$. Thus, when using the Skorokhod coupling lemma, we may also assume that $\sum_{k} k^{m} E_{t}(k) / n$ converges a.s. In particular, choosing $m=2, \sum_{k} k^{2} E_{t}(k) / n$ is a.s. bounded for each fixed rational $t$, and thus for each fixed $t>0$, since the sum is a decreasing function of $t$. This implies uniform summability of $k E_{s}(k) / n$, for any fixed $s>0$, and thus uniform summability in (4.14) and (4.23), by simple modifications of the arguments in Section 4; hence (4.14) and (4.23) hold for $s>0$. Similarly, $E_{s}(k) / n$ is uniformly summable for any fixed $s>0$, and (4.45) follows.

There are no other changes to the proofs of Theorems 1.1 and 1.2, since $\mu=\sum k p_{k}$ does not appear in the proof (except in a trivial way before (4.35)). We thus have the following result for random multigraphs.

Theorem 5.4. Let $\left(p_{k}\right)_{0}^{\infty}$ be a probability distribution. Assume that $n_{k} / n \rightarrow$ $p_{k}$ for each $k \in \mathbb{Z}^{+}$and that $\sum_{k=1}^{\infty} k n_{k} / n$ converges to a finite limit $\lambda$ as $n \rightarrow \infty$. Let $S_{\infty}^{(n)}$ denote the size of a random greedy independent set in the random multigraph $G^{*}\left(n,\left(d_{i}\right)_{1}^{n}\right)$.

Let $\tau_{\infty}$ be the unique value in $(0, \infty]$ such that

$$
\lambda \int_{0}^{\tau_{\infty}} \frac{e^{-2 \sigma}}{\sum_{k} k p_{k} e^{-k \sigma}} d \sigma=1
$$

Then

$$
\frac{S_{\infty}^{(n)}}{n} \rightarrow \lambda \int_{0}^{\tau_{\infty}} e^{-2 \sigma} \frac{\sum_{k} p_{k} e^{-k \sigma}}{\sum_{k} k p_{k} e^{-k \sigma}} d \sigma \quad \text { in probability. }
$$

Moreover, for each $k=0,1, \ldots$,

$$
\frac{S_{\infty}^{(n)}(k)}{n} \rightarrow \lambda \int_{0}^{\tau_{\infty}} e^{-2 \sigma} \frac{p_{k} e^{-k \sigma}}{\sum_{j} j p_{j} e^{-j \sigma}} d \sigma \quad \text { in probability. }
$$


Example 5.5. For an extreme example, let $d_{1}=n-1$ and $d_{2}=\cdots=d_{n}=$ 1 , i.e., there is one vertex of degree $n-1$ and all others have degree 1 . The limiting distribution is concentrated at 1: $p_{k}=\delta_{k 1}$ so $\mu=1$, but the average vertex degree $\sum_{k} k n_{k} / n=2(1-1 / n)$ which converges to $\lambda=2>\mu$.

Theorem 1.1 applies. The equation (1.2) is $\lambda \int_{0}^{\tau_{\infty}} e^{-\sigma} d \sigma=1$, and thus $\tau_{\infty}=\log 2$. Then (1.3) yields, with convergence in probability,

$$
\frac{S_{\infty}}{n} \rightarrow s_{\infty}=\lambda \int_{0}^{\tau_{\infty}} e^{-2 \sigma} d \sigma=2 \int_{0}^{\log 2} e^{-2 \sigma} d \sigma=\frac{3}{4} .
$$

This can also be seen directly. W.h.p., $G^{*}\left(n,\left(d_{i}\right)_{1}^{n}\right)$ consists of a star with centre at vertex $1, \approx n / 2$ leaves and $\approx n / 4$ loops at the centre, together with $\approx n / 4$ isolated edges. W.h.p., one of the leaves goes off before the centre of the star, and then the centre is blocked and the greedy independent set will actually be of maximum size, containing all the leaves of the star and one vertex from each isolated edge, together $\approx 3 n / 4$ vertices.

Note that only the multigraph version of the theorem applies in this case. The random simple graph $G\left(n,\left(d_{i}\right)_{1}^{n}\right)$ is deterministic and is a star with $n-1$ leaves. W.h.p, the first vertex that goes off is a leaf; then the centre is blocked and the greedy independent set will consist of all the leaves. Thus $S_{\infty}=n-1$ w.h.p. in the simple graph case.

Finally, we consider the case where $\lambda=\infty$. In this case, our formulae are no longer meaningful, but we can give at least a partial description of the outcome of the process via a different argument. In the case where $\sum_{k} p_{k}=1$, the result below implies that, w.h.p., almost all the vertices end up in the independent set, i.e., the jamming constant $s_{\infty}=1$.

Theorem 5.6. Suppose that $\lambda_{n}=\sum_{k} k n_{k} / n \rightarrow \infty$. Let $r(n)$ be the number of vertices of degree at most $\min \left(\lambda_{n}^{1 / 8}, n^{1 / 6}\right)$. Then, for every $\delta>0, S_{\infty} \geq$ $(1-\delta) r(n)$ w.h.p.

Proof. By considering subsequences, we may assume that $n_{0} / n \rightarrow p_{0}$ for some $p_{0} \in[0,1]$. The case $p_{0}=1$ is trivial, so we assume $p_{0}=\lim _{n \rightarrow \infty} n_{0} / n<$ 1.

Let $\varepsilon=\varepsilon(n)=\max \left(\lambda_{n}^{-1 / 7}, n^{-1 / 5}\right) \rightarrow 0$, and note that $\varepsilon^{6} \lambda_{n} / \log (1 / \varepsilon)$ and $\varepsilon^{4} n$ both tend to infinity.

Our first aim is to show that, at time $\varepsilon$, w.h.p., there are at most $3 \varepsilon \lambda_{n} n$ half-edges incident with empty vertices. The total number of half-edges incident with empty vertices of degrees up to $\varepsilon \lambda_{n}$ is at most $\varepsilon \lambda_{n} n$, so we turn our attention to vertices of larger degree.

Let $\tau_{1}$ be the first time that there are fewer than $\varepsilon^{3} n$ empty vertices of degree at least $\varepsilon \lambda_{n}$. Let $\tau_{2}$ be the time when the $\left\lceil\frac{1}{4} \varepsilon^{5} \lambda_{n} n\right\rceil$-th edge is paired. We claim that (a) $\tau_{1} \leq \tau_{2}$ w.h.p., and (b) $\tau_{1} \wedge \tau_{2} \leq \varepsilon$ w.h.p. Together these two imply that $\tau_{1} \leq \varepsilon$ w.h.p. 
To show (a), note that, as in the proof of Lemma 5.1, for every vertex $v$ of degree $k \geq \varepsilon \lambda_{n}$,

$$
\mathbb{P}\left(v \text { is empty at time } \tau_{2}\right) \leq\left(1-\frac{k}{\lambda_{n} n}\right)^{\frac{1}{4} \varepsilon^{5} \lambda_{n} n} \leq e^{-\frac{1}{4} \varepsilon^{6} \lambda_{n}}=o\left(\varepsilon^{3}\right) .
$$

Hence the expected number of empty vertices of degree at least $\varepsilon \lambda_{n}$ at time $\tau_{2}$ is $o\left(\varepsilon^{3} n\right)$, and the probability that there are as many as $\varepsilon^{3} n$ such vertices is $o(1)$.

To show (b), note that, before time $\tau_{1}$, vertices of degree at least $\varepsilon \lambda_{n}$ go off at a rate of at least $\varepsilon^{3} n$; when any such vertex goes off, at least $\frac{1}{2} \varepsilon \lambda_{n}$ edges are paired. For $0 \leq t \leq \tau_{1}$, let $P_{t}$ be the number of edges paired by time $t$. Then there is a Poisson process $Q_{t}$ of intensity $\varepsilon^{3} n$ such that $P_{t} /\left(\frac{1}{2} \varepsilon \lambda_{n}\right)$ dominates $Q_{t}$ for $t \leq \tau_{1}$, and

$$
\mathbb{P}\left(Q_{\varepsilon}<\frac{1}{2} \varepsilon^{4} n\right)=o(1)
$$

This implies that, w.h.p., either $\tau_{1}<\varepsilon$ or $P_{\varepsilon} \geq \frac{1}{4} \varepsilon^{5} \lambda_{n} n$, in which case $\tau_{2} \leq \varepsilon$. This completes the proof of (b).

We conclude that, w.h.p., $\tau_{1} \leq \varepsilon$, so that the number of empty vertices of degree at least $\varepsilon \lambda_{n}$ at time $\varepsilon$ is at most $\varepsilon^{3} n$. It follows that the number of half-edges incident with empty vertices of degrees between $\varepsilon \lambda_{n}$ and $\varepsilon^{-2} \lambda_{n}$ at time $\varepsilon$ is at most $\varepsilon \lambda_{n} n$.

It remains to consider empty vertices of degrees greater than $\varepsilon^{-2} \lambda_{n}$. For this range, we repeat the argument in Lemma 5.1. We take $n$ large enough that there are at least $\frac{1}{2}\left(1-p_{0}\right) n$ vertices of positive degree. Let $\tau_{\varepsilon}$ be the time when the $\left\lceil\frac{1}{9} \varepsilon\left(1-p_{0}\right) n\right\rceil$-th edge is created by a pairing. Until this time, there are always at least $\frac{1}{4}\left(1-p_{0}\right) n$ empty vertices of positive degree, and so the pairing rate is at least $\frac{1}{4}\left(1-p_{0}\right) n$. Thus

$$
\mathbb{P}\left(\tau_{\varepsilon}>\varepsilon\right) \leq \mathbb{P}\left(\operatorname{Po}\left(\frac{1}{4} \varepsilon\left(1-p_{0}\right) n\right)<\frac{1}{8} \varepsilon\left(1-p_{0}\right) n\right)=o(1) .
$$

Now, for each vertex $v$ of degree $k \geq \varepsilon^{-2} \lambda_{n}$,

$$
\mathbb{P}\left(v \text { is empty at time } \tau_{\varepsilon}\right) \leq\left(1-\frac{k}{\lambda_{n} n}\right)^{\frac{1}{9} \varepsilon\left(1-p_{0}\right) n} \leq e^{-\frac{1}{9} \varepsilon^{-1}\left(1-p_{0}\right)}=o(\varepsilon) .
$$

Thus the expected number of half-edges incident with empty vertices of degree at least $\varepsilon^{-2} \lambda_{n}$ at time $\tau_{\varepsilon}$ is $o\left(\varepsilon \lambda_{n} n\right)$. Hence, w.h.p., there are at most $\varepsilon \lambda_{n} n$ such half-edges at time $\varepsilon$.

Summing over the three ranges of degrees, we see that the number $U$ of half-edges incident with empty vertices at time $\varepsilon$ is w.h.p. at most $3 \varepsilon \lambda_{n} n$. The expected total number of half-edges incident with vertices that have gone off by time $\varepsilon$ is at most $\varepsilon \lambda_{n} n$, so w.h.p. at most $\frac{1}{2} \lambda_{n} n$ of the half-edges are paired by this time. Hence, w.h.p., there are at least $\frac{1}{2} \lambda_{n} n$ free halfedges at time $\varepsilon$, most of which are incident with vertices that have already become blocked. 
Now consider any vertex $v$ of degree $k \leq k_{0}$, where $k_{0}=k_{0}(n):=$ $\min \left(\lambda_{n}^{1 / 8}, n^{1 / 6}\right)=o\left(\varepsilon^{-1}\right)$. The probability that $v$ has gone off by time $\varepsilon$ is at most $\varepsilon$ and the probability that $v$ is blocked by time $\varepsilon$ is at most $k_{0} \varepsilon=o(1)$.

The conditional probability that $v$ will be blocked after time $\varepsilon$, given that $v$ has neither gone off nor has been blocked by time $\varepsilon$, and also given that $U \leq 3 \varepsilon \lambda_{n} n$ and there are at least $\frac{1}{2} \lambda_{n} n$ free half-edges at time $\varepsilon$, is at most the conditional probability that any of the half-edges incident with $v$ are later paired with a half-edge incident to another vertex that is empty at time $\varepsilon$, which is at most $k_{0} U / \frac{1}{2} \lambda_{n} n \leq 6 \varepsilon k_{0}=o(1)$. Thus the (unconditional) probability that $v$ will be blocked is $o(1)$ and $v$ ends up in the independent set with probability $1-o(1)$. Hence the expected number of vertices of degree at most $k_{0}$ that do not appear in $S_{\infty}$ is $o(r(n))$, and the result follows.

The function $\min \left(\lambda_{n}^{1 / 8}, n^{1 / 6}\right)$ can certainly be improved via an adjustment of the parameters in the argument, but it seems unlikely that this will give the best possible result. As we shall see in Example 5.8 below, some dependence on $n$ is essential.

We finish with two examples to illustrate some of the possible behaviours in this range.

Example 5.7. Fix constants $\alpha, \gamma>0$ with $2 / 3>\alpha>1 / 2+\gamma$.

Take a set $A$ of $n^{\alpha}$ vertices of degree $n^{\alpha}$, and a set $B$ of $n-n^{\alpha}$ vertices of degree $n^{\gamma}$. So all but a proportion $\approx n^{1+\gamma-2 \alpha} \ll n^{-\gamma}$ of the half-edges are incident with $A$, and so most vertices of $B$ have all their neighbours in $A$. We have $\lambda_{n}(n) \approx n^{2 \alpha-1}$, and the degrees of vertices in $B$ can be up to $\lambda_{n}^{1 / 2-\delta}$ for any $\delta>0$.

Now consider what happens when the first $n^{\alpha}$ vertices go off (including vertices that are already blocked when they go off). W.h.p., at most $2 n^{2 \alpha-1}$ of the vertices that go off among the first $n^{\alpha}$ are in $A$, and so at most $2 n^{3 \alpha-1}=o(n)$ vertices in $B$ become blocked as a result. Also at most $n^{\alpha+\gamma}=o(n)$ vertices in $B$ become blocked as a result of vertices in $B$ going off. Meanwhile, w.h.p., at least $\frac{1}{2} n^{\alpha}$ vertices in $B$ go off, and this generates at least $\frac{1}{4} n^{\alpha+\gamma}$ pairings, most of which are to vertices in $A$. The probability that a given vertex of $A$ is not blocked during this process is thus exponentially small.

So at this point, w.h.p., all vertices of $A$ are blocked, and almost all vertices of $B$ are empty, with all their neighbours blocked. Hence the independent set has size $(1-o(1))|B|=(1-o(1)) n$.

This example shows, among other things, that the jamming constant of the random multigraph can equal 1 even if all the $p_{i}$ are equal to 0 .

If we allow $\lambda_{n}$ to be extremely large, then there is an even greater variety of possible behaviour. In the example below, the size of the random greedy independent set is very far from being concentrated. 
Example 5.8. Let $A$ be a set of $n / 2$ vertices of degree $n^{1+\delta}$, for some $\delta>0$, and let $B$ be a set of $n / 2$ vertices of degree $n^{\beta}$, where $\beta \geq 3+5 \delta$. So $\lambda_{n}$ is approximately $\frac{1}{2} n^{\beta}$. Then, w.h.p., every vertex in $B$ is adjacent to every vertex of $A \cup B$, and no two vertices of $A$ are adjacent.

In this instance, if a vertex of $B$ is the first to go off, then $S_{\infty}=1$, while if a vertex of $A$ is the first to go off, then $S_{\infty}=n / 2$.

By making $\beta$ large, we can ensure that the degrees of vertices of $A$ can be an arbitrarily small power of $\lambda_{n}$.

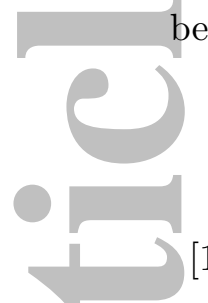

[1] M. Bayati, D. Gamarnik and P. Tetali, Combinatorial approach to the interpolation method and scaling limits in sparse random graphs, Ann. Prob. 41 (2013) 4080-4115.

[2] P. Bermolen, M. Jonckheere and P. Moyal, The jamming constant of random graphs. Preprint, 2013. arXiv:1310.8475

[3] B. Bollobás, A probabilistic proof of an asymptotic formula for the number of labelled regular graphs, Europ. J. Comb. 1 (1980) 311-316.

[4] B. Bollobás, The independence ratio of regular graphs, Proc. Amer. Math. Soc. 83 (1981) 433-436.

[5] A. Cadilhe, N.A.M. Araúyo and V. Privman, Random sequential adsorption: from continuum to lattice and pre-patterned substrates, $J$. Phys. Cond. Mat. 19 (2007), Article 065124.

[6] P.J. Diggle, Statistical Analysis of Spatial and Spatio-temporal Point Patterns, 3rd edition (2014), CRC Press, Boca Raton, FL, 2014.

[7] J. Ding, A. Sly and N. Sun, Maximum independent sets on random regular graphs, to appear in Acta Mathematica. arXiv:1310.4787

[8] J.W. Evans, Random and cooperative sequential adsorption, Rev. Mod. Phys. 65 (1993) 1281-1329.

[9] W. Feller, An Introduction to Probability Theory and Its Application, volume I, third edition, Wiley, New York, 1968.

10] S.R. Finch, Mathematical Constants, Encyclopedia of Mathematics and its Applications 94. Cambridge Univ. Press, Cambridge, 2003.

[11] P.J. Flory, Intramolecular reaction between neighbouring substituents of vinyl polymers, J. Am. Chem. Soc. 61 (1939) 1518.

[12] A.M. Frieze and T. Łuczak, On the independence numbers of random regular graphs, J. Combin. Theor. B 54 (1992) 123-132.

[13] A.M. Frieze and S. Suen, On the independence number of random cubic graphs, Rand. Struct. Alg. 5 (1994) 649-664.

[14] D. Gamarnik and D.A. Goldberg, Randomized greedy algorithms for matchings and independent sets in regular graphs: exact results and finite girth corrections, Comb. Prob. Comput. 19 (2010) 61-85.

[15] L. Gerin, The Page-Rényi parking process, Electronic J. Comb. 22 (2015), Paper P4.4. 
[16] S. Janson, The probability that a random multigraph is simple, Comb. Prob. Comput. 18 (2009) 205-225.

[17] S. Janson, The probability that a random multigraph is simple, II. J. Appl. Probab. 51A (2014) 123-137.

[18] S. Janson and M.J. Luczak, A simple solution to the $k$-core problem, Rand. Struct. Alg. 30 (2007) 50-62.

[19] S. Janson and M.J. Luczak, A new approach to the giant component problem, Rand. Struct. Alg. 34 (2009) 197-216.

[20] S. Janson, M.J. Luczak and P. Windridge, Law of large numbers for the SIR epidemic on a random graph with given degrees, Rand. Struct. Alg. 45 (2014) 724-761.

[21] O. Kallenberg, Foundations of Modern Probability Theory, second edition, Springer-Verlag, New York, 2002.

[22] J. Lauer and N.C. Wormald, Large independent sets in regular graphs of large girth, J. Combin. Theor. B 97 (2007) 999-1009.

[23] C. McDiarmid, Colouring random graphs, Annals of Operations Research 1 (1984) 183-200.

[24] E.S. Page, The distribution of vacancies on a line, J. R. Statist. Soc. B 21 (1959) 364-374.

[25] M.D. Penrose, Random packing, sequential adsorption and the jamming limit, Commun. Math. Phys. 218 (2001) 153-176.

[26] A. Rényi, On a one-dimensional random space-filling problem, Publ. Math. Res. Inst. Hung. Acad. Sci. 3 (1958) 109-127.

[27] N.C. Wormald, Differential equations for random processes and random graphs, Ann. Appl. Prob. 5 (1995) 1217-1235.

[28] N.C. Wormald, Models of random regular graphs, in Surveys in Combinatorics 1999, London Mathematical Society Lecture Note Series.

Department of Mathematics, London School of Economics, Houghton Street, LONDON WC2A 2AE, UNITED KINGDOM

E-mail address: g.r.brightwell@lse.ac.uk

URL: http://www.maths.lse.ac.uk/Personal/graham/

Department of Mathematics, Uppsala University, PO Box 480, SE-751 06

UPPSALA, SWEDEN

E-mail address: svante.janson@math.uu.se

URL: http://www. math.uu.se/ svante/

School of Mathematics, Queen Mary, University of LONDON

E-mail address: m.luczak@qmul.ac.uk

$U R L:$ http://www . maths.qmul.ac.uk/ luczak/ 


\section{University Library}

\section{- M M N E R VA A gateway to Melbourne's research publications}

Minerva Access is the Institutional Repository of The University of Melbourne

Author/s:

Brightwell, G;Janson, S;Luczak, M

Title:

The Greedy Independent Set in a Random Graph with Given Degrees

Date:

2017-12-01

Citation:

Brightwell, G., Janson, S. \& Luczak, M. (2017). The Greedy Independent Set in a Random Graph with Given Degrees. RANDOM STRUCTURES \& ALGORITHMS, 51 (4), pp.565-586. https://doi.org/10.1002/rsa.20716.

Persistent Link:

http://hdl.handle.net/11343/292875 